\title{
Efficacy of L2 on L3 segment perception: a case study on native Arabic speakers' discriminability of Korean vowels
}

\author{
Seung-ah Hong \\ Department of Korean language and literature, Hankuk University of Foreign \\ Studies, Republic of Korea \\ https://doi.org/10.36505/ExLing-2012/05/0016/000222
}

\begin{abstract}
The study aims to investigate cross-linguistic influence in speech perception by tracing the source of L3 vowel perception. Two groups of native Egyptian Arabic speakers, monolinguals and late-bilinguals of English, were examined for their ability to discriminate Korean vowels. It was assumed that the discriminability depends on the factors such as L2 experience, phonological constraint of L1, type of memory, and phonetic contrasts. The result suggests that L2 experience could enhance sensitivity to certain L3 vowel contrasts. However, L2 experience does not do much to overcome an L1 phonological constraint. Also, it was found that even in the phonemic process, listeners may rely on auditory memory for discriminating the novel speech sounds.
\end{abstract}

Key words: Korean, Arabic, vowel, perception, L3

\section{Introduction}

It has been well-acknowledged that listeners' L1 phonology greatly affects the acquisition of L2 phonology. In terms of the L2 segment perception, listeners perceive L2 segments based on the reflection of L1 phoneme inventory. That is, if a phonological contrast which is not available in L1 exists in L2, listeners have difficulty in perceiving it (i.e., Perception of /r/ vs. /l/ by Japanese speakers). However, even if a contrast is available in the listeners' L1 phonology, which can also trigger phonemic distinction in L2, listeners may face the difficulty in perceiving it due to a phonological constraint in their L1 (i.e., perception of word-final English stops by German speakers). Analogous to these, dialectal variation within a language shall be noticed as individual languages since it can also lead the perceptual differences in perceiving L2 speech sounds (see Escudero \& Williams 2012). Likewise, in many ways, L1 shows very robust effects to L2 speech perception. However, when it comes to an additional language (L3), that a listener has a prior experience in L2, it becomes more complicated since the speech perception of the latter may be involved not only with listeners' L1, but L2 phonology (See Williams \& Hammarberg 1998). There is still a question of what is the source for L3 speech perception.

Answering this question, this study examined listeners' discrimination ability of novel speech sounds (L3: Korean vowel) based on their L2 experience (L1: Egyptian Arabic, L2: English). Since Arabic vowel

ExLing 2012: Proceedings of 5th Tutorial and Research Workshop on Experimental Linguistics, 27-29 August 2012, Athens, Greece 
inventory is far smaller than English and Korean ${ }^{1}$, it was hypothesized that Arabic speakers with English learning experience (GB) would grasp the phonetic contrasts in Korean vowels better than the monolinguals (GM) so the discriminability of GB would be higher than GM. Also, Arabic phonology allows $\mathrm{CV}$ as its standard syllable unit while English accepts the $\mathrm{V}$ as a basic syllable unit; it was assumed that GM would perform better when the vowels were delivered in $\mathrm{CV}$ than in $\mathrm{V}$ while it would not matter for GB listeners.

\section{Methodology}

The participants were 11 Cairene EA speakers (Mean age of 35). Since the phonology of colloquial Arabic differs between each dialect, the native Arabic speakers in this study were restricted to Egyptian Arabic speakers born and raised in Cairo. They were divided into two groups (GM and GB) based on their learning experiences of English (L2): monolinguals (6 in GM) vs. late-bilingual of English (5 in GB). The English proficiency of GB listeners may differ from each other, but all were at the level that they could communicate with native English speakers while the participants in GM could not understand ever a simple word in English.

Seven Korean vowels $(/ \mathrm{a} / / \mathrm{l} / /, \mathrm{u} /, / \mathrm{e} /, / \mathrm{o} /, / \wp /$, and $/ \mathrm{i} /)$, in two different syllable contexts (V and $h \mathrm{~V}$ ) were used as stimuli. Each vowel was paired up based on the proximity in the vowel space, thus eight sets of contrasts /a$\wp /, / \mathrm{a}-\mathrm{e} /, / \mathrm{i}-\mathrm{e} /, / \mathrm{i}-\mathrm{i} /, / \mathrm{u}-\mathrm{o} / / \mathrm{u}-\mathrm{i} / / / \mathrm{o}-\wp /$ and $/ \mathrm{i}-\wp /$ were made. The pairs of vowels consist of stimuli in ABX discrimination task with 1500ms-ISI. Participants were guided to choose one odd stimulus out of three. The test trials were composed of 8 contrasts, 2 syllable contexts, 4 set types (AAB, ABB, BAA, BBA), 3 times of iteration, and control trials (AAA, BBB) were also added in order to see the reliability of the experiment. In total, there were 234 trials.

\section{Results}

Logit-loglinear analysis was employed to analyze the result since the nature of the data is frequency data, and it was required to see how each factor (L2 experience: L, syllable context: $S$, type of memory: $M$, and phonetic contrast: P) attributes to the result. Therefore, the null hypothesis is that all variables interact together as an effect of vowel distinction, and this can be represented as the model as $\{$ LSMP $\}$. If there can be an another model which consists of fewer variables but still fits the saturated model, the variable that is missed in the model is a non-factor and can be neglected. The goal of this analysis is to find the simplest model that does not reject the null hypothesis.

As a result, $\{\mathrm{LP}\}\{\mathrm{SP}\}\{\mathrm{T}\}$ was selected as the best model (See Table1) $)^{2}$. That is, all four variables were factors that decide the discriminative capacity 
of Korean vowels. However, not all of them attribute independently to the result, and they rather associate with each other in a way that: L2 experiences improve only the discriminability of certain phonetic contrasts $\{\mathrm{LP}\}$, however it was unrelated to the disciminability under different syllable contexts or the target stimulus position. Similarly, the vowels delivered in $h \mathrm{~V}$ were more beneficial to listeners than in $\mathrm{V}$ for discrimination in general, but not in all cases $\{\mathrm{SP}\}$. Although the experiment was set that the listeners go through the phonemic process, the performance of both GM and GB showed the recency effect under any type of syllable contexts and contrasts, and this means that the listeners relied on auditory sensitivity for judgment regardless of the condition (see Best et al. 2001).

Table 1. Result of hierarchical models by logit-loglinear analysis.

\begin{tabular}{|l|l|l|l|}
\hline \hline Model & df & L2 & P \\
\hline$\{$ LSM $\},\{$ LSP $\},\{$ LMP $\},\{$ SMP $\}$ & 11.688 & 7 & 0.111 \\
\hline$\{$ LP $\},\{$ SP $\},\{$ M $\}$ & 35.450 & 39 & 0.633 \\
\hline$\{$ SP $\},\{$ TC $\}$ & 42.415 & 40 & 0.367 \\
\hline$\{$ SP $\},\{L\},\{$ M $\}$ & 47.140 & 46 & 0.426 \\
\hline$\{$ SP $\},\{M\}$ & 50.011 & 47 & 0.355 \\
\hline
\end{tabular}

\section{Discussion}

The findings posits that the source of L3 speech sounds can be flexible in between listeners' L1 or L2 since it shows that the L2 experience not only helps to improve the sensitivity towards L3 segments, but it could also possibly deteriorate the sensitivity or to have no affect at all. This may be related to the robustness of established L2 category. As in Flege's SLM (e.g., Flege et al. 1997), if an L2 category is established successfully due to the absence of such phonetic category in listeners' L1, the established L2 category could be later used as the source for relevant L3 speech sounds. Also, it is presumed that L1 phonological constraints are stronger than L2 rules; although segmental properties of novel speech sounds were acquired, they could be still governed by the rules in L1.

\section{Notes}

1. Modern Standard Arabic is known as that it has three vowels, both long and short vowels of /a/, /i/, and /u/ (Al-Ani 1970). Here, /e:/, /o:/ were added up for Cairene Egyptian Arabic (Becker 2010). Although colloquial Arabic shows more of allophonic variation in of vowels, its vowel inventory is still smaller than English

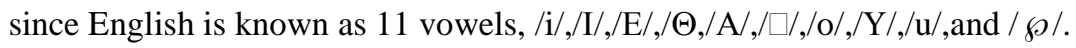

2. The models in Table 2 are all satisfied as the saturated model \{LSMP\}. Then, the model such as $\{\mathrm{SP}\}\{\mathrm{M}\}$ could be the best one among them. However, when the residual cells were reviewed, the other models include some cases which are not 
explainable. The model $\{\mathrm{LP}\}\{\mathrm{SP}\}\{\mathrm{M}\}$ was the only one without any unexplainable case, and therefore, it was selected as the best model.

\section{Acknowledgements}

This study is supported by Korea Research Foundation with the Global $\mathrm{PhD}$ fellowship.

\section{References}

Al-Ani, S. 1970. Arabic Phonology: An Acoustical and Physiological Investigation. The Hague, Mouton.

Becker, R. 2010. Roy Becker's vowel corpus. Retrieved from http://www.linguistics.ucla.edu/faciliti/facilities/databases/databases.html

Best, C., McRoberts, G. and Goodell, E. 2001. Discrimination of non-native consonant contrasts varying in perceptual assimilation to the listener's native phonological system. Journal of the Acoustical Society of America 109, 775-794.

Escudero, P. and Williams, D. 2011. Perceptual assimilation of Dutch vowels by Peruvian Spanish listeners. Journal of the Acoustical Society of America 129, 1-7.

Flege, J., Bohn, O., and Jang, S. 1997. The effect of experience on nonnative subjects' production and perception of English vowels. Journal of Phonetics 25, 437-470.

Williams, S. and Hammarberg, B. 1998. Language switches in L3 production: Implications for a polyglot speaking model. Applied Linguistics 19, 295-333. 